\title{
Control of rubidium low-lying Rydberg states with trichromatic femtosecond $\pi$-pulses for ultrafast quantum information processing
}

\author{
I. J. Afa, J. L. Font, C. Serrat \\ UPC - Universitat Politècnica de Catalunya, Colom 11, 08222 Terrassa (Barcelona), Spain*
}

(Dated: October 28, 2017)

\begin{abstract}
We propose an ultrafast femtosecond time scale trichromatic $\pi$-pulse illumination scheme for coherent excitation and manipulation of low-lying Rydberg states in rubidium. Selective population of $\mathrm{nP}_{3 / 2}$ levels with principal quantum numbers $\mathrm{n} \lesssim 12$ using 75 fs laser pulses is achieved. The density-matrix equations of a four-level ladder system beyond the rotating-wave approximation have to be solved to clarify the balance between the principal quantum numbers, the duration of the laser pulses and the associated ac-Stark effects for the fastest optimal excitation. The mechanism is robust for femtosecond control using different level configurations for applications in ultrafast quantum information processing and spectroscopy.
\end{abstract}

PACS numbers:

\section{INTRODUCTION}

Selective excitation and manipulation of Rydberg states plays a crucial role in quantum information processing, a field of rapid theoretical and experimental progress with a large number of proposals for quantum information processors being investigated $[1,2]$. A central issue is the design of fast quantum gates, with recent proposals using neutral atoms and molecules that allow gate operation times set by the time scale of the laser excitation [3-5]. In the case of neutral atoms, the requirement that the gate operation time is short compared to the typical time of decoherence mechanisms, including spontaneous emission, collisions, ionization of the Rydberg states, transitions induced by black body radiation, or motional excitation of the atoms trapped in an optical lattice, leads to the search of state-selective Rydberg excitation schemes using femtosecond pulses. Despite the fact that excitation to a single n-Rydberg level requires nanosecond or $\mathrm{cw}$ lasers with a narrow bandwidth, coherent control tools as control algorithms to optimally shape femtosecond laser pulses have been successfully used to address a single transition [6,7], as well as multipulse schemes using 150 fs pulses, which alone would populate about ten n-Rydberg levels, have shown the ability to selectively populate a single or a few levels $[8,9]$. These different schemes address the excitation of relatively high Rydberg levels, typically of principal quantum numbers as $n \sim 30$. Several models have recently been proposed however to implement fast quantum gates in neutral atoms by using specific low-lying Rydberg states $[3,4]$, for which faster femtosecond time scale selective coherent excitation schemes are demanded.

Studies on coherent population trapping (CPT) and electromagnetically induced transparency (EIT) set the basis for many coherent control techniques based on combined detuned lasers and adiabatic transfer [10-16]. The

*Electronic address: carles.serrat-jurado@upc.edu concept of CPT was employed to demonstrate pumping of atomic systems in a particular state, and has been theoretically analyzed by Arimondo and Orriols [11] and Gray et al. [12] based on the solution of the Bloch-like equations of motion for a 3-level system with applications in Sodium atoms. This concept was further experimentally demonstrated by Whitley and Stroud [14]. The main goal of these studies was to optimally pump as much population as possible to the excited state from the ground state with the requirements of a proper combination of density matrix (DM) relaxation rates, Rabi frequencies and precise detuning of the laser field. Previous studies employing adiabatic transfer schemes have been used to demonstrate EIT for achieving population transfer to bounded states in atomic systems [13, 17, 18] and molecular systems $[19,20]$, with extensions to solid media [21]. Some studies also showed the possibility of extending these techniques to four-level schemes, achieving a limited but significant amount of population transfer [22].

This previous research showed the potential for other interesting phenomena in multilevel systems, such as transfer to higher bound and continuum levels [12], and development of techniques for efficient transfer to unpopulated Rydberg states [19], which is essential for further advancement on spectroscopy, collision dynamics, quantum memories [21] and photon control in quantum information processing [23]. Kuklinski et al. [19] showed an extension to the Heisenberg picture which presents a solution of the Liouville equations that allows the computation of the relaxation processes during the evolution of population transfer in multilevel systems. Zhang and George [24-26] employed the dynamical solution of the Liouville equations to find a practical path to more efficient CPT in Fullerenes with a proper combination of parameters, like time delay and pulse duration.

CPT and EIT have also been demonstrated in Rubidium with extensions to different multilevel configurations, for instance, in a 3 -level $\Lambda$-type and ladder-type atomic systems in ${ }^{87} \mathrm{Rb}$ atoms cooled and confined in a magnetooptical trap [27], in a three-level V-type system in a $\mathrm{D}_{2}$ 
transition of ${ }^{87} \mathrm{Rb}$ [28], in a 4-level inverted-Y atomic system [29], and in a 4-level tripod atomic system in the ${ }^{87} \mathrm{Rb} \mathrm{D}_{1}$ line [30].

The present study analyses a particular control scheme based on the Rabi flopping behavior involving polychromatic $\pi$-pulses. According to the area theorem, a fully resonant $\pi$-pulse represents a field with a 180 -degree rotation of its pseudopolarization vector on the Bloch sphere. Assuming a two level atomic system with all population in the ground state, illumination by a $\pi$-pulse will drive the atom to the excited state due to a $\pi$-rotation on the Bloch sphere, resulting in complete population inversion [31-36]. For ultrafast control, $\pi$-pulse polychromatic control techniques benefit from the fact that no delay between pulses is involved as compared to adiabatic schemes, so that the interaction time remains of the order of the laser pulse temporal width. The control of population transfer between a pair of quantum states involving single or polychromatic transitions by illuminating the atom with resonant $\pi$-pulses has extensively been implemented using from long to ultrashort pulses $[32,37,38]$. For the case of a four-level ladder system, population transfer schemes combining adiabatic and $\pi$ pulse techniques have also been proposed [39].

Optimal $\pi$-pulse single photon excitation from the ground state to a low-lying Rydberg level with ultrashort femtosecond pulses is not feasible due to the small dipole coupling coefficients associated to UV transitions, which require a large electric field to produce a $\pi$ area and result in ac-Stark splittings from all other allowed transitions, which frustrates the $\pi$-pulse excitation. In the present paper, we show that this impediment can be circumvented by using trichromatic $\pi$-pulse excitation, an approach that was recently reported as a robust method for complete population inversion in a four-level system [38]. We show that the higher values of the dipole coupling coefficients associated to the transitions in a four-level scheme allow for ultrafast excitation of low-lying Rydberg states, without the need of deep UV wavelengths and with field intensities that avoid significant ac-Stark effects.

We explore the possibilities of trichromatic $\pi$-pulse control for ultrafast selective excitation and manipulation of low-lying Rydberg states in rubidium $(\mathrm{Rb})$, as a paradigmatic system for most experiments with Rydberg atoms. Figure 1 illustrates the basic control mechanisms of trichromatic $\pi$-pulse excitation in a four-level ladder system, as it was also reported in [38]. From left to right in Fig. 1, complete population inversion from level 1 to level 4 is produced by simultaneous illumination of the atom with a set of pulses with areas as $\sqrt{3} \pi, 2 \pi$, and $\sqrt{3} \pi$, which corresponds to the effect of a $\pi$-pulse in a two-level system. The intermediate levels 2 and 3 become temporally populated during the process and become completely de-populated at the end of the interaction. Complete de-excitation of the atom is accomplished by the same set of pulses, which is shown by the central interaction in Fig. 1, while a two-level like $2 \pi$-pulse is

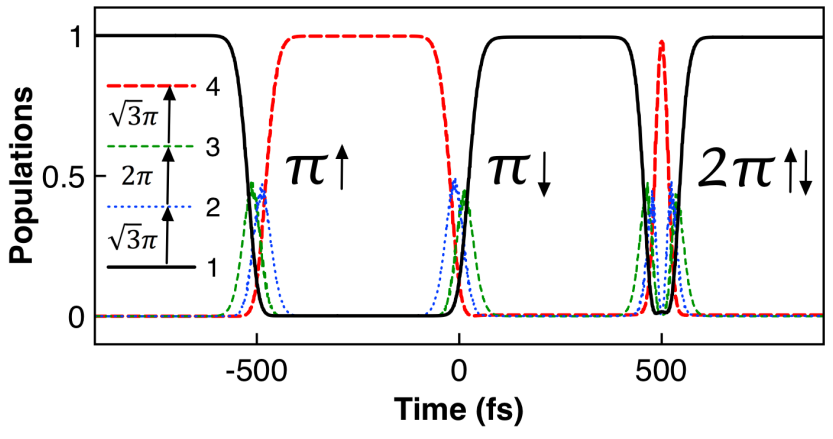

FIG. 1: (color online) In analogy to the effect of a resonant $\pi$-pulse in a two-level system, which completely transfers the population from one state to the other, the figure schematically shows the effect of a resonant "trichromatic $\pi$-pulse" in a four-level ladder system. A set of 75 fs pulses with areas $\sqrt{3} \pi$, $2 \pi$, and $\sqrt{3} \pi$, each one resonant with a particular transition as indicated, completely transfer the population from the lowest to the highest state (up- $\pi$-pulse, left), or in the other direction (down- $\pi$-pulse, center). Using "trichromatic $2 \pi$-pulses", this is of areas $2 \sqrt{3} \pi, 4 \pi$, and $2 \sqrt{3} \pi$, the system is completely excited and then de-excited, as shown by the third interaction process in the figure (up-and-down- $2 \pi$-pulse, right).

produced with doubling the areas of the three pulses.

Different requirements have to be considered for the success of the trichromatic $\pi$-pulse scheme for ultrafast excitation of Rydberg states. On the one hand, the energies of the chosen atomic transitions have to be different enough compared to the spectral bandwidth of the laser pulses, so that each individual pulse interacts only with its corresponding transition. On the other hand, since the area $A$ of the pulses is fixed, with $A \propto \mu \tau E$, the product $\mu \tau$ of the electric dipole moment coefficients $\mu$ and the pulses duration $\tau$ has to be sufficiently large so that the envelope field $E$ does not produce important ac-Stark effects. The spectral separation between adjacent levels in the low-lying Rydberg states region in Rb is suitable to accommodate the bandwidth of femtosecond pulses for optimal selective excitation, and as it will be shown in our calculations, the dipole moment coefficients in a four-level ladder scheme warrant its implementation for ultrafast complete population transfer in a trichromatic $\pi$-pulse configuration.

The paper is organized as follows: in Section II the mathematical model and the main results from the simulations are presented. In Section III we discuss the influence of the transition dipole moments together with the pulse durations. In Section IV we further show the advantages of using $\pi$-pulse multiphoton processes versus single-photon excitations. In Section $\mathrm{V}$ we discuss the need of avoiding the RWA in our simulations by comparing the results with the ones obtained in the RWA, and end with the conclusions. 


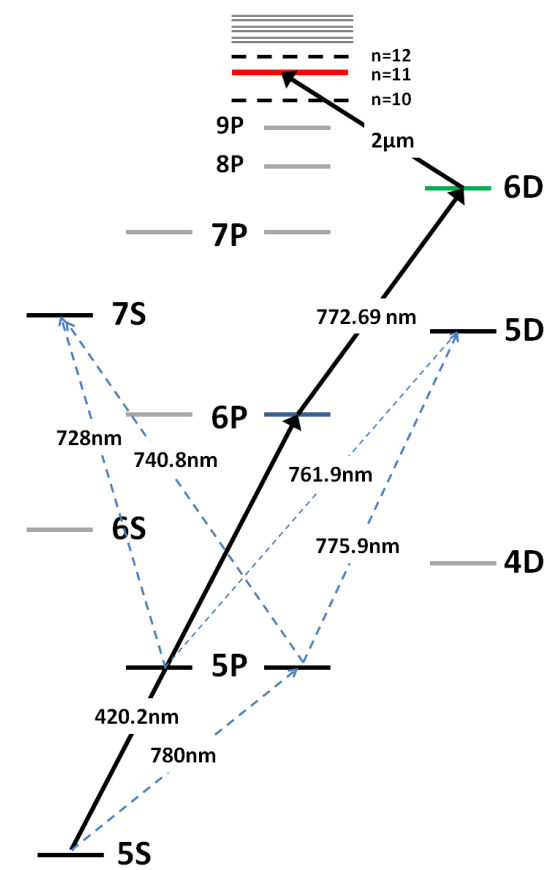

FIG. 2: (color online) Energy levels configuration of the Rb atom chosen to excite the low-lying Rydberg states $\mathrm{nP}_{3 / 2}$ with principal quantum numbers $\mathrm{n} \lesssim 12$.

\section{MODEL AND SIMULATIONS}

A 4-level cascade configuration has been chosen in the present study for Rubidium atoms as shown in Fig. 2. As it will come up from the simulations below, our results can be extended however to other level configurations and other systems. The ground state is the $5 \mathrm{~S}_{1 / 2}$ level of $\mathrm{Rb}$ (level 1), which is coupled with a $420.2 \mathrm{~nm}$ laser pulse to the $6 \mathrm{P}_{3 / 2}$ (level 2), with $\mu_{12}=0.54 \times 10^{-29}$ $\mathrm{Cm}$. The laser pulse has an area of $\sqrt{3} \pi$ [38]. The $6 \mathrm{P}_{3 / 2}$ level is coupled to the $6 \mathrm{D}_{3 / 2}$ (level 3) with a $772.69 \mathrm{~nm}$ laser pulse, with $\mu_{23}=1.01 \times 10^{-29} \mathrm{Cm}$. This laser pulse has an area of $2 \pi$. The precise value of the $3-4$ transition energy does not vary the results, so that in our simulations we safely consider a wavelength of $2 \mu \mathrm{m}$ for the transition from $6 \mathrm{D}_{3 / 2}$ to the $\mathrm{nP}_{3 / 2}$ Rydberg state (level 4). The laser pulse coupling this transition has an area of $\sqrt{3} \pi$. The value of $\mu_{34}$ sets the quantum number $\mathrm{n}$ of level 4 and it is varied in our study in the range $0.25 \times 10^{-29}<\mu_{34}<1.25 \times 10^{-29} \mathrm{Cm}$. The precise values for the dipole matrix coefficients have been taken from the data in $[41,42]$, from which we also estimate $\mu_{14}=0.02 \times 10^{-29} \mathrm{Cm}$ for the allowed $5 \mathrm{~S}_{1 / 2} \rightarrow \mathrm{nP}_{3 / 2}$ transition, considering $\mathrm{n} \sim 11$.

We evaluate the balance between the principal quantum number $\mathrm{n}$ of the Rydberg states available for excitation and the duration of the pulses for optimal selective population inversion by trichromatic $\pi$-pulse excitation in the femtosecond time scale. For pulse durations $\lesssim 500$ fs only decoherence processes of the order or faster than picoseconds can affect the coherent interaction. The natural decay times associated to a four-level system as in Fig. 2 are in the nanosecond and microsecond time scales and they can be neglected [40]. Collision and other possible broadening mechanisms are examined in our study by comparing the results for pure dephasing times as short as $T_{2}=1 \mathrm{~ns}$ and $T_{2}=1 \mathrm{ps}$. We obtain robust almost $100 \%$ selective excitation of the $(\mathrm{n} \lesssim 12) \mathrm{P}_{3 / 2}$ levels of $\mathrm{Rb}$ with 75 fs pulses with pure dephasing of $1 \mathrm{~ns}$, and $\approx 95 \%$ population transfer for 1 ps.

The density-matrix equations of the four-level system have to be considered without the assumptions of the rotating-wave approximation (RWA), as it will be further discussed below. The equations read

$$
\begin{aligned}
& \dot{\rho}_{22}=i \frac{E(t)}{\hbar}\left(\mu_{12}\left(\rho_{12}-\rho_{21}\right)+\mu_{23}\left(\rho_{32}-\rho_{23}\right)\right) \\
& \dot{\rho}_{33}=-i \frac{E(t)}{\hbar}\left(\mu_{23}\left(\rho_{32}-\rho_{23}\right)+\mu_{34}\left(\rho_{34}-\rho_{43}\right)\right) \\
& \dot{\rho}_{44}=i \frac{E(t)}{\hbar}\left(\mu_{14}\left(\rho_{14}-\rho_{41}\right)+\mu_{34}\left(\rho_{34}-\rho_{43}\right)\right) \\
& \dot{\rho}_{11}=-\dot{\rho}_{33}-\dot{\rho}_{22}-\dot{\rho}_{44} \\
& \dot{\rho}_{12}=-\Gamma_{12} \rho_{12} \\
& +i \omega_{12} \rho_{12}+i \frac{E(t)}{\hbar}\left(\mu_{12}\left(\rho_{22}-\rho_{11}\right)+\mu_{14} \rho_{24}^{*}-\mu_{23} \rho_{13}\right) \\
& \dot{\rho}_{13}=-\Gamma_{13} \rho_{13} \\
& +i \omega_{13} \rho_{13}+i \frac{E(t)}{\hbar}\left(\mu_{12} \rho_{23}-\mu_{23} \rho_{12}+\mu_{14} \rho_{34}^{*}-\mu_{34} \rho_{14}\right) \\
& \dot{\rho}_{14}=-\Gamma_{14} \rho_{14} \\
& +i \omega_{14} \rho_{14}+i \frac{E(t)}{\hbar}\left(\mu_{14}\left(\rho_{44}-\rho_{11}\right)-\mu_{34} \rho_{13}+\mu_{12} \rho_{24}\right) \\
& \dot{\rho}_{23}=-\Gamma_{23} \rho_{23} \\
& +i \omega_{23} \rho_{23}+i \frac{E(t)}{\hbar}\left(\mu_{23}\left(\rho_{33}-\rho_{22}\right)-\mu_{34} \rho_{24}+\mu_{12} \rho_{13}\right) \\
& \dot{\rho}_{24}=-\Gamma_{24} \rho_{24} \\
& +i \omega_{24} \rho_{24}+i \frac{E(t)}{\hbar}\left(\mu_{12} \rho_{14}-\mu_{14} \rho_{12}^{*}+\mu_{23} \rho_{34}-\mu_{34} \rho_{23}\right) \\
& \dot{\rho}_{34}=-\Gamma_{34} \rho_{34} \\
& +i \omega_{34} \rho_{34}+i \frac{E(t)}{\hbar}\left(\mu_{34}\left(\rho_{44}-\rho_{33}\right)+\mu_{23} \rho_{24}-\mu_{14} \rho_{13}^{*}\right)
\end{aligned}
$$

where $\rho_{i i}$ are the populations of the levels $i$, the offdiagonal elements $\rho_{i j}$ represent the coherences between levels $i, j, \mu_{i j}$ are the dipole coupling coefficients of the corresponding transitions, and $\omega_{i j}=\left(\epsilon_{j}-\epsilon_{i}\right) / \hbar$ are their angular frequencies, with $\epsilon_{i}$ being the energies of the corresponding states. The relaxation of the coherences are considered by pure dephasing rates $\Gamma_{i j}=T_{2}^{-1}$. The laser pulses are of Gaussian shape, with the electric field $E(t)$ given by

$$
\begin{aligned}
& E(t)=E_{1}(t)+E_{2}(t)+E_{3}(t) \\
& =e^{-(t / \tau)^{2}}\left[E_{01} \cos \left(\omega_{1} t\right)+E_{02} \cos \left(\omega_{2} t\right)+E_{03} \cos \left(\omega_{3} t\right)\right],
\end{aligned}
$$


where $E_{01}, E_{02}$ and $E_{03}$ are the peak amplitudes of the three pulse components $E_{1}(t), E_{2}(t)$ and $E_{3}(t)$, and $\omega_{1}$, $\omega_{2}$ and $\omega_{3}$ are the optical angular frequencies coupling the transitions $1-2,2-3$, and $3-4$, respectively. The duration of the pulses is given by $\tau=t_{p} /(\sqrt{2 \ln 2})$, where $t_{p}$ is the full width at half maximum of the pulse intensity profile. In the present simulations all pulses are considered of the same duration.
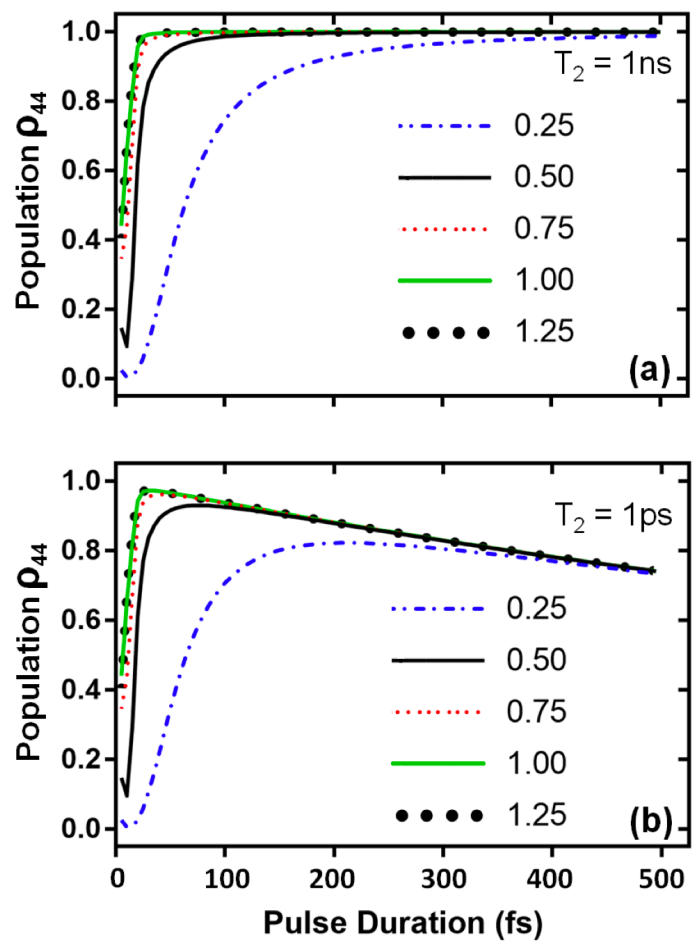

FIG. 3: (color online) Final population of the excited Rydberg state $\left(\rho_{44}\right)$ as a function of pulse duration, for dephasing times (a) $T_{2}=1 \mathrm{~ns}$ and (b) $T_{2}=1 \mathrm{ps}$, and for $\mu_{34}=0.25,0.50$, $0.75,1.00,1.25$, as indicated, in units of $10^{-29} \mathrm{Cm}$.

\section{INFLUENCE OF DIPOLE MOMENT AND PULSE DURATION}

As discussed above, the selection of pulse duration plays a crucial role in achieving selective manipulation of the desired Rydberg state [43]. Figure 3 shows the final population of level 4 that is obtained by trichromatic $\pi$-pulse illumination of the system, this is, by simultaneously sending a set of resonant pulses with areas $\sqrt{3} \pi$ in the first transition $\left(5 \mathrm{~S}_{1 / 2} \rightarrow 6 \mathrm{P}_{3 / 2}\right), 2 \pi$ in the second transition $\left(6 \mathrm{P}_{3 / 2} \rightarrow 6 \mathrm{D}_{3 / 2}\right)$, and $\sqrt{3} \pi$ in the third transition $\left(6 \mathrm{D}_{3 / 2} \rightarrow \mathrm{nP}_{3 / 2}\right)$. As discussed above and as shown in Fig. 1, for sufficiently long $\mu \tau$ products and negligible decoherence this illumination scheme completely transfers the population from the ground state $5 \mathrm{~S}_{1 / 2}$ to the Rydberg state $\mathrm{nP}_{3 / 2}$. The final population $\rho_{44}$ is eval- uated in Fig. 3 as a function of the pulses duration for $t_{p}<500 \mathrm{fs}$, and for different values of the $\mu_{34}$ transition dipole coupling coefficient. Figure 3 (a) shows the results for a pure dephasing of 1 ns and Fig. 3 (b) considers a faster dephasing of 1 ps. We observe that, in both cases, for laser pulse durations shorter than about $25 \mathrm{fs}$, the population transfer to the highest state (level 4) is strongly suppressed. This can be explained by the acStark effect produced in the different transitions of the system, which can only be properly computed by considering the density-matrix equations beyond the RWA. With fixed pulse areas, such short pulses $\left(t_{p} \lesssim 25\right.$ fs) involve high electric fields that produce significant ac-Stark shifts which destroy the trichromatic $\pi$-pulse population inversion process. As shown in Fig. 3 (a), population transfer to level 4 is not effective either for a transition dipole value as small as $\mu_{34}=0.25 \times 10^{-29} \mathrm{Cm}$ and for pulse durations $t_{p} \lesssim 300 \mathrm{fs}$, which is due to the same effect i.e. the electric field in the 3-4 transition becomes too large. Since the $\mu_{34}$ transition dipole coupling coefficient decreases as the Rydberg state quantum number $\mathrm{n}$ increases, the value of $\mu_{34}$ eventually sets the highest $\mathrm{nP}_{3 / 2}$ Rydberg state that can be optimally accessed for a given duration of the laser pulses. This is clear for dephasings of $1 \mathrm{~ns}$ as shown in Fig. 3 (a) and also for the 1 ps dephasing in Fig. 3 (b). Fig. 3 (b) shows how the fast 1 ps dephasing degrades the population transfer as the duration of the pulses increases, as it can be expected since the dephasing time becomes shorter than the full interaction time.

As mentioned above, our goal is to selectively populate the highest $\mathrm{nP}_{3 / 2}$ Rydberg state with the shortest femtosecond pulse. The results in Fig. 3 suggest to consider a value of the dipole moment larger than approximately $\mu_{34}=0.5 \times 10^{-29} \mathrm{Cm}$, which corresponds to the $(\mathrm{n} \lesssim 12) \mathrm{P}_{3 / 2}$ low-lying region of the Rydberg states in $\mathrm{Rb}[41,42]$. To be selective in the excitation process, we further need a spectral bandwidth of the pulses sufficiently narrow compared to the separations between the levels in the region that we are considering, which are $702 \mathrm{~cm}^{-1}$ for $9 \mathrm{P}_{3 / 2}-10 \mathrm{P}_{3 / 2}, 460 \mathrm{~cm}^{-1}$ for $10 \mathrm{P}_{3 / 2}-$ $11 \mathrm{P}_{3 / 2}$, and about $300 \mathrm{~cm}^{-1}$ for $11 \mathrm{P}_{3 / 2}-12 \mathrm{P}_{3 / 2}$. Therefore, our simulations show that laser pulses of about 75 fs, which have a linewidth of $195.7 \mathrm{~cm}^{-1}$, will produce the desired ultrafast selective population inversion process from the $5 \mathrm{~S}_{1 / 2}$ ground state to the $(\mathrm{n} \lesssim 12) \mathrm{P}_{3 / 2}$ low-lying region of Rydberg states in Rb. A further impediment for the success of the ultrafast process is the possible weak coupling of the used laser pulses to other non-resonant transitions of the system. For instance, in the present case, the ground state $5 \mathrm{~S}_{1 / 2}$ couples to the $5 \mathrm{P}_{3 / 2}$ level with $780 \mathrm{~nm}$ (see Fig. 2), which is close to the $772.69 \mathrm{~nm}$ laser used in our scheme but is still sufficiently a part to be strongly involved. A system with a larger number of levels than the four-level system studied here should be used to consider the quantitative effect of all close transitions. Nevertheless, these undesirable nonresonant couplings would ultimately be reduced by using 
some longer laser pulses. Population transfer from level 1 to level 4 in more than $99 \%$ is hence achieved with 75 fs pulses and $\mu_{34}=0.5 \times 10^{-29} \mathrm{Cm}(\mathrm{n} \sim 11)$ for $1 \mathrm{~ns}$ dephasing [Fig. 3(a)], which is reduced to $\approx 95 \%$ in the case of the fast 1 ps dephasing [Fig. 3(b)]. This optimal case is also shown in Fig. 4.

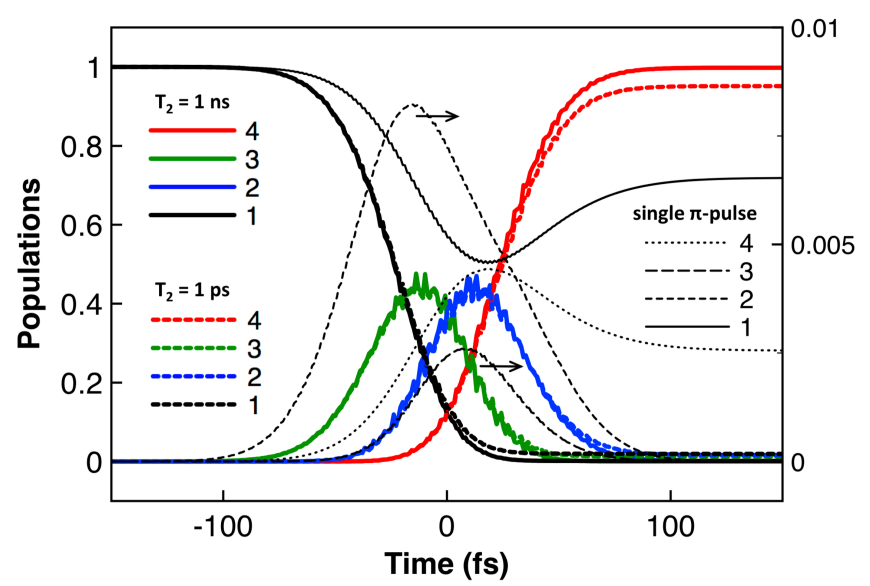

FIG. 4: (color online) Time evolution of the populations for the interaction with 75 fs laser pulses with $\mu_{34}=0.5 \times 10^{-29}$ Cm ( $\mathrm{n} \sim 11)$, for $T_{2}=1 \mathrm{~ns}$ and $T_{2}=1 \mathrm{ps}$, as indicated. The black-thin lines (legend on the right) show the case of illumination with a single pulse of area $\pi$ resonant with the 1-4 transition, considering $\mu_{14}=0.02 \times 10^{-29} \mathrm{Cm}$ and $T_{2}=1$ ns. Note that levels 2 and 3 become slightly populated during the interaction (right $\mathrm{y}$-axis).

\section{SINGLE $\pi$-PULSE VERSUS MULTIPHOTON $\pi$-PULSE SCHEMES}

In this section, we show the advantage of using a multiphoton $\pi$-pulse over a single $\pi$-pulse scheme for driving the population to the targeted Rydberg state. Figure 4 shows the time evolution of the four populations for optimal illumination with 75 fs laser pulses with $\mu_{34}=0.5 \times 10^{-29} \mathrm{Cm}$, and for dephasing times of $1 \mathrm{~ns}$ and 1 ps. The resulting peak intensities of the optimal 75 fs pulses with $\sqrt{3} \pi, 2 \pi$, and $\sqrt{3} \pi$ areas are $1.17 \times 10^{11}$ $\mathrm{W} / \mathrm{cm}^{2}, 0.44 \times 10^{11} \mathrm{~W} / \mathrm{cm}^{2}$ and $1.37 \times 10^{11} \mathrm{~W} / \mathrm{cm}^{2}$, respectively. As required for optimal population inversion, partial population and depopulation of the intermediate levels is not affected by spontaneous emission during the process for these pulse durations, which is advantageous compared to other three-photon excitation schemes with narrower linewidth lasers [44-49]. As also shown in Fig. 4 , although most population is transferred to level 4 at the ending of the pulses, where the field intensity is low, at time about $20 \mathrm{fs}-$ which is within the FWHM of a 75 fs Gaussian pulse - the population in the final Rydberg state is already $\sim 50 \%$, and therefore ionization from the Rydberg states might weakly affect the process. A quantitative study of the effect of ionization would however depend on the particular conditions and geometry [50] and it is beyond the scope of the present analysis.

The black-thin lines in Fig. 4 show the evolution of the populations in the case of illuminating the system with a single 75 fs $\pi$-pulse resonant with the UV 1-4 transition $\left(5 \mathrm{~S}_{1 / 2} \rightarrow 11 \mathrm{P}_{3 / 2}\right)$. Considering the dipole coupling coefficient $\mu_{14}=0.02 \times 10^{-29} \mathrm{Cm}[41,42]$, the peak intensity of the $\pi$-pulse results in $2.85 \times 10^{13} \mathrm{~W} / \mathrm{cm}^{2}$. In a two-level system (i.e. if levels 2 and 3 were not present), this resonant $\pi$-pulse would produce complete population inversion from level 1 to level 4. However, due to the effect of the pulse on the intermediate levels in the four-level system, the $\pi$-pulse complete population inversion is frustrated by the ac-Stark shifts produced in the non-resonant transitions involving levels 2 and 3 . The coupling of the pulse with the other transitions is apparent in Fig. 4 (right y-axis) since levels 2 and 3 become slightly populated during the interaction. The ac-Stark shift produced by a field of peak amplitude $|E|$ resonant with the 1-4 transition on the 1-2 transition, which is far from resonance, is given by [51]

$$
\Delta \omega=\frac{\mu_{12}|E|}{2 \hbar}\left(\frac{1}{\omega_{12}+\omega_{14}}+\frac{1}{\omega_{12}-\omega_{14}}\right),
$$

which gives $-160.6 \mathrm{~cm}^{-1}$ for the single $75 \mathrm{fs} \pi$-pulse. This frequency shift of the transition is hence of the order of the linewidth of the 75 fs laser pulse $\left(195.7 \mathrm{~cm}^{-1}\right)$ and, as it was also discussed in [38], it prevents optimal $\pi$-pulse population inversion (see black-thin lines in Fig. 4). For a higher value of dipole coupling in the 1-4 transition, such as $\mu_{14} \gtrsim 0.1 \times 10^{-29} \mathrm{Cm}$, the resulting electric field is already weak to substantially affect the system $(\lesssim 1.14 \times$ $\left.10^{12} \mathrm{~W} / \mathrm{cm}^{2}\right)$, in this case $|\Delta \omega| \lesssim 6.42 \mathrm{~cm}^{-1}$ and the $\pi$-pulse complete inversion is recovered. It is therefore clear how the single $\pi$-pulse population inversion is not effective for a direct ultrashort UV excitation from $5 \mathrm{~S}_{1 / 2}$ to $\mathrm{nP}_{3 / 2}$.

\section{DISCUSSION AND CONCLUSIONS}

The results that we have obtained with the spectrally wide 75 fs pulses hence involve Fourier components that substantially interact out of resonance with the dipole allowed transitions of the system, and therefore it becomes necessary to consider the density matrix equations beyond the RWA. Figure 5 shows a comparison of the simulations performed without the RWA with simulations performed considering the RWA. In the RWA the fields are coupled only to their respective transitions, and therefore the effect on the other transitions is absent, so that complete inversion is obtained for all pulse durations [Fig. 5 (a)]. The disagreement between the two theories is clear 

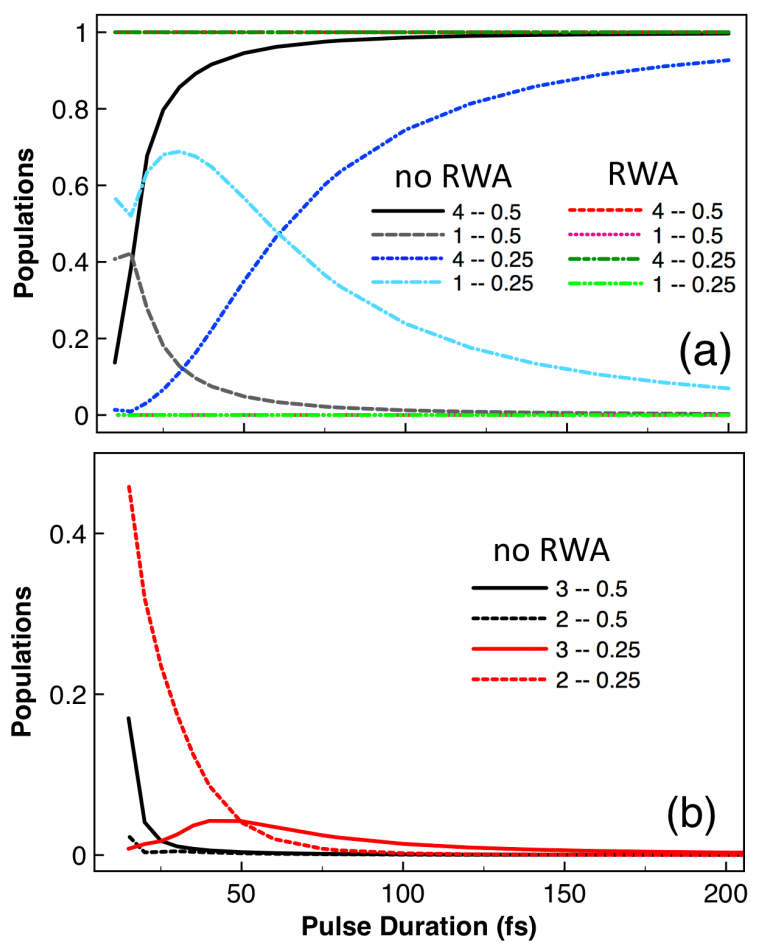

FIG. 5: (color online) Comparison of the calculations without and with the RWA. (a) The final populations of levels 1 and 4 are shown, considering trichromatic $\pi$-pulse excitation and the level configuration of Fig. 2 , for $\mu_{34}=0.5$ and 0.25 , as indicated - in units of $10^{-29} \mathrm{Cm}$, and considering $T_{2}=1$ ns. (b) Final populations of levels 2 and 3 without the RWA. Levels 2 and 3 become empty using the RWA in all cases.

for pulses shorter than $\sim 100$ fs for $\mu_{34}=0.5 \times 10^{-29}$
Cm. For $\mu_{34}=0.25 \times 10^{-29} \mathrm{Cm}$ the results converge for durations $\gtrsim 500 \mathrm{fs}$ [see also Fig. 3(a)]. Figure 5 (b) shows how the coupling of the pulse with the other transitions results in population left in levels 2 and 3 after the interaction, which is only seen without the RWA.

Summing up, the inapplicability of single-photon UV excitation to reach and control Rydberg states with femtosecond laser pulses leads to the need of using multiphoton processes. We demonstrate ultrafast selective population of rubidium low-lying Rydberg states using trichromatic $\pi$-pulse excitation. 75 fs laser pulses with areas such as $\sqrt{3} \pi, 2 \pi$, and $\sqrt{3} \pi$ produce purely coherent excitation of $(\mathrm{n} \lesssim 12) \mathrm{P}_{3 / 2}$ Rb Rydberg states due to the short time scale involved in the interaction. Our study shows that some higher-n Rydberg states might also be reached by using the same technique but with some longer pulses, which would also reduce the possible non-resonant couplings with other transitions involved in the system. Therefore, although we have chosen a particular configuration of $\mathrm{Rb}$, the fundamental mechanism can readily be extended to other configurations and using different geometries. To the best of our knowledge, this is the fastest scheme that has been proposed for coherent excitation and manipulation of low-lying Rydberg states, with applications in quantum information technology and high precision spectroscopy.

\section{Acknowledgments}

Support from the Spanish Ministry of Economy and Competitiveness through FIS2014-51997-R is acknowledged.
[1] M. Saffman and T. G. Walker, "Quantum information with Rydberg atoms", Rev. Mod. Phys. 82, 2313 (2010).

[2] M. Saffman, "Quantum computing with atomic qubits and Rydberg interactions: progress and challenges", J. Phys. B: At. Mol. Opt. Phys. 49, 202001 (2016).

[3] D. Jaksch, J. I. Cirac, and P. Zoller, S. L. Rolston, R. Côté, M. D. Lukin, "Fast Quantum Gates for Neutral Atoms", Phys. Rev. Lett. 85, 2208 (2000).

[4] L.-M. Duan, "Scaling Ion Trap Quantum Computation through Fast Quantum Gates", Phys. Rev. Lett. 93, 100501 (2004).

[5] R. de Vivie-Riedle and U. Troppmann, "Femtosecond Lasers for Quantum Information Technology", Chem. Rev. 107, 5082 (2007).

[6] J. Ahn, T. C. Weinacht, and P. H. Bucksbaum, Science 287, 463 (2000).

[7] T. C. Weinacht, J. Ahn, and P. H. Bucksbaum, Nature (London) 397, 233 (1999).

[8] J. Preclíková, M. Kozák, D. Fregenal, Ø. Frette, B. Hamre, B. T. Hjertaker, J. P. Hansen, and L. Kocbach, "Excitation of Rydberg wave packets with chirped laser pulses", Phys. Rev. A 86, 063418 (2012).
[9] M. Kozák, J. Preclíková, D. Fregenal, J. P. Hansen, "State-selective Rydberg excitation with femtosecond pulses", Phys. Rev. A 87, 043421 (2013).

[10] E. Arimondo, Progress in Optics (Amsterdam: Elsevier) 35, 257 (1996).

[11] E. Arimondo, G. Orriols, "Nonabsorbing atomic coherences by coherent two-photon transitions in a three-level optical pumping.", Lett. Nuovo Cimento 17, 333 (1976).

[12] H. R. Gray, R. M. Whitley, C. R. Stroud, "Coherent trapping of atomic populations.", Opt. Lett. 3, 218 (1978).

[13] S. Harris, "Electromagnetically Induced Transparency" Phys. Today 50, 36 (1997).

[14] R.M. Whitley, C.R. Stroud, "Double optical resonance" Phys. Rev. A 14, 1498 (1976).

[15] N. V. Vitanov, A. A. Rangelov, B. W. Shore, K. Bergmann, "Stimulated Raman adiabatic passage in physics, chemistry, and beyond.", Rev. Mod. Phys. 89, 015006 (2017).

[16] M.O. Scully and M.S. Zubairy, Quantum Optics, Cambridge University Press, Cambridge 2006.

[17] Y. Li, M. Xiao, "Transient properties of an electromagnetically induced transparency in three-level atoms" 
Phys. Rev. A 51, R2703 (1995).

[18] K.J. Boller, A. Imamoglu, S. Harris, "Observation of electromagnetically induced transparency" Phys. Rev. Lett. 66, 2593 (1991).

[19] J.R. Kuklinski, U. Gaubatz, F.T. Hioe, K. Bergmann, "Adiabatic population transfer in a three-level system driven by delayed laser pulses" Phys. Rev. A 40, 6741 (1989).

[20] U. Gaubatz, P. Rudecki, S. Schiemann, K. Bergmann, "Population transfer between molecular vibrational levels by stimulated Raman scattering with partially overlapping laser fields. A new concept and experimental results" J. Chem. Phys. 92, 5363 (1990).

[21] M. Fleischbauer, A. Imamoglu, J.P. Marangos, "Electromagnetically induced transparency: Optics in coherent media" Rev. Mod. Phys. 77, 633 (2005).

[22] Y.B. Band, P.S. Julienne, "Population transfer by multiple stimulated Raman scattering" J. Chem. Phys. 95, 5681 (1991).

[23] M.D. Lukin, A. Imamoglu, "Controlling photons using electromagnetically induced transparency" Nature 413, 273 (2001).

[24] G.P. Zhang, T. George, "Proposed coherent trapping of a population of Electrons in a C 60 molecule induced by laser excitation" Phys. Rev. Lett. 109, 257401 (2012).

[25] G.P. Zhang, T. George, "Laser-induced coherent population trapping in C 60" Phys. Rev. A 88, 063808 (2013).

[26] G.P. Zhang, "Hidden identity in a generic $\Lambda$ system: applications to coherent population trapping" J. Phys. B: At. Mol. Opt. Phys. 46, 035504 (2013).

[27] M. Yan, E.G. Rickey, Y. Zhu, "Electromagnetically induced transparency in cold rubidium atoms" J. Opt. Soc. Am. B 18, 1057 (2001).

[28] B.C. Das, D. Bhattacharyya, A. Das, S. Chakrabarti, S. De, "Spontaneous observation of electromagnetically induced transparency (EIT) and absorption (EIA) in a multi-level V-type system of ${ }^{87} \mathrm{Rb}$ and theoretical simulation of the observed spectra using a multi-mode approach" J. Chem. Phys. 145, 224312 (2016).

[29] J. Kou, G. Wan, Z.H. Kang, H.H. Wang, L. Jiang, X.J. Zhang, Y. Jiang, J.Y. Gao, "EIT-assisted large crossKerr nonlinearity in a four-level inverted-Y atomic system" J. Opt. Soc. Am. B 27, 2035 (2010).

[30] X. Yang, S. Li, C. Zhang, H. Wang, "Enhanced crossKerr nonlinearity via electromagnetically induced transparency in a four-level tripod atomic system" J. Opt. Soc. Am. B 26, 1423 (2009).

[31] L. Allen, J.H. Eberly, Optical Resonance and Two-level Atoms Dover Publication, New York 1987.

[32] J.-C. Diels and W. Rudolph, Ultrashort Laser Pulse Phenomena Fundamentals, Techniques, and Applications on a Femtosecond Time Scale (Second Edition), Elsevier 2006.

[33] X. Chen, I. Lizuain, A. Ruschhaupt, D. Guery-Odelin, J.G. Muga, "Shortcut to Adiabatic Passage in Two- and Three-level Atoms" Phys. Rev. Lett. 105, 123003 (2010).

[34] M. Holthaus, B. Just, "Generalized $\pi$ pulses" Phys. Rev. A 49, 1950 (1994).

[35] G.N. Gibson, "Multiphoton $\pi$ pulses" Phys. Rev. Lett.
89, 263001 (2002).

[36] J. Oreg, G. Hazak, J.H. Eberly, "Multilevel inversion schemes in and beyond the adiabatic limit" Phys. Rev. A 32, 2776 (1985).

[37] C. Serrat and J. Biegert, "Energy efficient method for two-photon population transfer with near-resonant chirped pulses", Opt. Express 16, 19667 (2008).

[38] C. Serrat, M. Corbera, J. Afa, "Trichromatic $\pi$-Pulse for Ultrafast Total Inversion of a Four-Level Ladder System", Appl. Sci. 5, 1484 (2015).

[39] B. Zhang, J-H Wu, X-Z Yan, L. Wang, X-J Zhang, JY Gao, "Coherence generation and population transfer by stimulated Raman adiabatic passage and $\pi$ pulse in a four-level ladder system", Opt. Express 1912000 (2011).

[40] A. E. Siegman, Lasers, University Science Books, Sausalito CA 94965 (1986), p. 99.

[41] M. S. Safronova, W. R. Johnson, and A. Derevianko, "Relativistic many-body calculations of energy levels, hyperfine constants, electric-dipole matrix elements, and static polarizabilities for alkali-metal atoms", Phys. Rev. A 60, 4476 (1999).

[42] M. S. Safronova, Carl J. Williams, and Charles W. Clark, "Relativistic many-body calculations of electric-dipole matrix elements, lifetimes and polarizabilities in rubidium", Phys. Rev. A 69, 022509 (2004).

[43] X.-H. Li, X.-Z. Zhang, R.-H. Zhang, X.-D. Yang, "Population transfer via adiabatic passage in Rydberg potassium atom", Chinese Phys. 16, 2924 (2007).

[44] T. Vogt, M. Viteau, J. Zhao, A. Chotia, J. Zhao, D. Comparat, and P. Pillet, "Electric-field induced dipole blocakde with Rydberg atoms", Phys. Rev. Lett. 97, 083003 (2006).

[45] J. Deiglmayr, M. Reetz-Lamour, T. Amthor, S. Westermann, A. L. de Oliviera, M. Weidemuller, "Coherent excitation of Rydberg atoms in an ultracold gas", Opt. Comm. 264, 298 (2006).

[46] P. Thoumany, T. Germann, T. Hansch, G. Stania, L. Urbonas, and T. Becker, "Spectroscopy of rubidium Rydberg states with three diode lasers", J. Mod. Opt. 56, 2055 (2009).

[47] I. I. Ryabtsev, I. I. Beterov, D. B. Tretyakov, V. M. Entin, E. A. Yakshina, "Doppler- and recoil-free laser excitation of Rydberg states via three-photon transitions", Phys. Rev. A 84, 053409 (2011).

[48] J. Qian, J. Zhai, L. Zhang, W. Zhang W, "Chirped multiphoton adiabatic passage for four-level ladder-type Rydberg excitation", Phys. Rev. A 91, 013411 (2015).

[49] I. I. Ryabtsev, I. I. Beterov, D. B. Tretyakov, V. M. Entin, E. A. Yakshina, "Spectroscopy of cold rubidium Rydberg atoms for applications in quantum information", Physics - Uspekhi 59, 196 (2016).

[50] E. A. Volkova, A. M. Popov, O. V. Tikhonova, "Numerical modeling of the photoionization of Rydberg atoms by the field of an electromagnetic wave", J. Exp. Theor. Phys. 86, 328 (1998).

[51] S. H. Autler and C. H. Townes," Stark Effect in Rapidly Varying Fields", Phys. Rev. 100, 703 (1955). 